\title{
A New Simple Radiological Classification Measuring the Height of the Tegmen Tympani
}

\author{
Sherif Idris ${ }^{1}$, Youness Elkhalidy ${ }^{1}$, Ravi Bhargava ${ }^{2}, \mathrm{DABR}^{2}$ and Allan $\mathrm{Ho}^{1}$ \\ ${ }^{1}$ Division of Otolaryngology-Head and Neck Surgery, Department of Surgery, Canada
}

${ }^{2}$ Department of Radiology and Diagnostic Imaging, University of Alberta Hospital, Canada

Submission: June 21, 2018; Published: July 16, 2018

*Corresponding author: Youness Elkhalidy, Division of Otolaryngology-Head and Neck Surgery, Department of Surgery, Canada, Tel: 7809645036; Email: elkhalid@ualberta.ca

\begin{abstract}
Objective: The objective of this study was to examine the height of the tegmen tympani with respect to the lateral semicircular canal in patients undergoing computed tomographic assessment as part of their work-up for sensorineural hearing loss.

Design: High resolution computed tomography scans of the temporal bones of 62 patients who underwent imaging as part of their otologic work-up for sensorineural hearing loss were analyzed. A total of 100 radiographically normal temporal bones were assessed and the distance between the lateral semicircular canal and the lowest point of the tegmen tympani was measured in both the coronal and sagittal planes.

Results: The distance between the lateral semicircular canal and the lowest point of the tegmen tympani in both the coronal and sagittal planes display a unimodal but slightly right-skewed distribution. The coronal values did not significantly deviatiate from a normal distribution $(\mathrm{p}=0.203)$ with mean \pm standard deviation of $4.4 \mathrm{~mm} \pm 1.2 \mathrm{~mm}$. In the sagittal plane, analysis using Kolmogorov-Smirnov test showed no significant deviation from the truncated normal distribution $(\mathrm{p}=0.11$ ) with a mean \pm standard deviation of $2.7 \mathrm{~mm} \pm 1.4 \mathrm{~mm}$.

Conclusion: The height of the tegmen tympani at its lowest point with respect to the lateral semicircular canal follows a unimodal normal distribution. We propose using the lateral semicircular canal as a landmark in the classification of the tegmen tympani during surgical planning with computed tomography. The lateral semicircular canal is a useful stable radiological landmark, which is distinct and not often obliterated by diseases of the temporal bone.

Keywords: Tegmen Tympani; High Resolution Computed Tomography; Scoring System; Lateral Semicircular Canal

Abbreviations: HRCT: High Resolution Computed Tomography; LSCC: Lateral Semicircular Canal; SSC: Superior Semicircular Canal; EAC: External Auditory Canal; CSF: Cerebrospinal Fluid; DICOM: Digital Imaging and Communication in Medicine; SNHL: Sensorineural Hearing Loss; SD: Standard Deviation; IQR: Interquartile Range
\end{abstract}

\section{Introduction}

Radiologic imaging can be a useful tool in the evaluation of individuals with hearing loss, particularly when surgical intervention is being considered as part of the treatment plan. The ability of high resolution computed tomography (HRCT) to accurately assess the structures of the temporal bone represents a major advancement in the pre-operative evolution of patients with pathology to the temporal bone [1]. HRCT is often used to evaluate diseases of the middle ear and mastoid, such as cholesteatoma, and may better inform the physician regarding possible anatomical variations and the extent of the underlying disease process [2]. Additionally, imaging results may be used by some physicians to enhance the informed consent process prior to surgery, whilst discussing the risks of potential complications. HRCT is generally used as the first imaging modality because of its superior bony definition [1]. In particular HRCT can be used for evaluation of the tegmen and surrounding osseous structures when planning middle ear operations. The tegmen is a very thin layer of bone that serves as the roof of the mastoid and tympanic cavity, separating it from the middle cranial fossa. The shape of the tegmen demonstrates marked anatomical variability between individual patients and even between temporal bones of the same patient. These dimensional variations must be taken into account when planning surgery in the mastoid and middle ear space, particularly when mastoidectomy is being considered, as complications may arise when operating near the tegmen. Injury to the tegmen and dura may result in intracranial complications which include subdural hematoma, cerebrospinal fluid (CSF) leakage, and cerebral parenchymal damage [3]. Risk factors for dural complications include low placement of the tegmen, unusual tegmen shape, type and extent of middle ear and mastoid disease, and the level of experience of the surgeon [4]. Because the tegmen may be lower than expected in certain individuals, it is important that such situations with an increased risk of surgical complications be determined in the preoperative period. This study examines the height of the tegmen tympani in the sagittal and coronal planes relative to the lateral semicircular canal (LSCC) in patients with normal temporal bones undergoing work-up for sensorineural and/or conductive hearing loss. 


\section{Global Journal of Otolaryngology}

\section{Methods}

\section{Study Design}

The study was approved by the ethics review board at the University of Alberta and was conducted at the University of Alberta Hospital, a tertiary care otologic referral center. This was a retrospective observational study of consecutive patients receieving HRCT scanning of the temporal bones as part of their work-up for sensorineural and/or conductive hearing loss.

\section{Ethical Considerations}

As a retrospective observational study, our main ethical concerns involved protecting patient sensitive information. We therefore removed all patient identifying data from radiographic images and spread sheets in order to assure confidentiality. We also processed and stored all of our data onsite using a password protected computer in the radiology department. Ethics was obtained through the Research Ethics Office at the University of Alberta with the protocol number Pro00033880.

\section{Patients}

The study examined 62 consecutive patients who underwent HRCT scanning of the temporal bones as part of their otologic assessment for senorineural hearing loss between January 2013 and January 2014. Imaging was performed to aid in determining the etiology of the SNHL and to detect or rule out congenital, infectious, inflammatory, or tumoural pathology. None of the patients had a documented history of chornic ear disease. Patients were excluded if they were less than 18 years of age or had previous temporal bone surgery. In patients with unilateral disease of the temporal bones, we excluded the diseased side and included the normal contralateral side in our final data analysis.

\section{CT Scan Acquisition and Measurements}

HRCT scans of the temporal bones were acquired at The University of Alberta Hospital on a 128 slice SOMATOM Definition Flash scanner (Siemens, Munich, Germany) using the following CT parameters: single tube spiral acquisition at $120 \mathrm{kV}, 400$ effective mAs, ultra-high-resolution filter, and $0.6 \mathrm{~mm}$ section thickness. Digital Imaging and Communication in Medicine
(DICOM) images were retrieved and any identifying patient information was removed to maintain patient confidentiality. Each individual patient scan consisted of separate imaging series for both the right and the left temporal bones in the axial and coronal planes. Measurements in the coronal plane were made on images where the slice thickness was $0.6 \mathrm{~mm}$. Coronal plane imaging data was imported onto a PACS work-station (Impax 6.6; Agfa HealthCare, Greenville, South Carolina). Images were reconstructed into axial planes parallel through the plane of the lateral semicircular canal using an Aquarius Workstation v4.4 (TeraRecon, San Mateo, California). The plane that traversed both tubes of the lateral semicircular canal was chosen as a reference plane and projected into the ipsilateral middle ear cavity. Two perpendicular planes, one in the parasagittal plane and one in the paracoronal plane were reconstructed along the midpoint of the reference plane within the middle ear cavity. We chose to measure the lowest point of the tegmen tympani on the paracoronal slice where the scutum, Prussak's space, and incus were present. The distance between the roof of the middle ear cavity, along 5 equally placed points within the middle ear cavity, and the horizontal plane subtended from the lateral semicircular canal was measured on this chosen paracoronal slien. This measurement defined our variable of interest in the coronal plane - the height in millimiters from the LSCC to the lowest point on the tegmen tympani $\left(h_{c}\right)$. Parasagittal images were generated perpendicular to the paracoronal plane and the reference plane subtended from the horizontal place through the lateral semicircular canals on the Aquarius Workstation. from the original data with Measurements in the parasagittal plane were made on images where the slice thickness was $1 \mathrm{~mm}$. The lowest point of the tegmen tympani with regards to the previously created LSCC plane was then measured on this computer generated parasagittal image (Figure 1A-1C). This measurement defined our variable of interest in the sagittal plane - the height in millimiters from the LSCC to the lowest point on the tegmen tympani $\left(h_{s}\right)$. Statistical tests (mean, median, and standard deviation) were calculated for the lowest measurements of the tegmen tympani height in both the coronal $\left(h_{c}\right)$ and the sagittal $\left(h_{s}\right)$ planes.
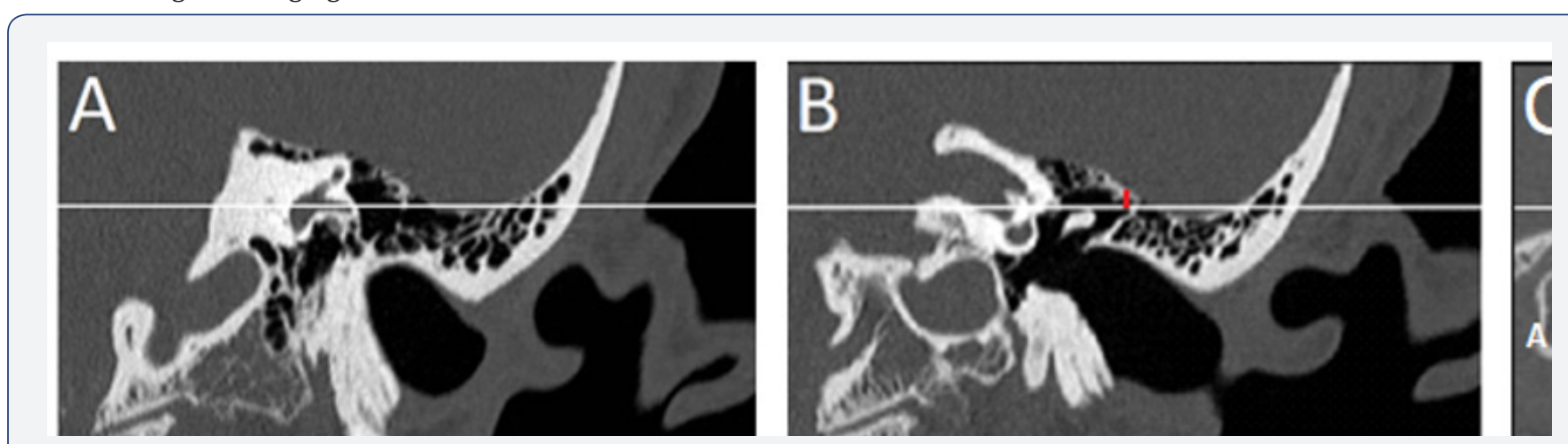

Figure 1: A. Horizontal line (white) through the plane of the lateral semicircular canal on a coronal image. B. Vertical line (red) perpendicular to the plane of the lateral semicircular canal used to measure the lowest coronal height of the tegmen tympani $\left(h_{c}\right)$. C. Horizontal line through the plane of the lateral semicircular from the coronal images. Vertical linerepresenting the lowest sagittal height of the tegmen tympani $\left(h_{s}\right)$. $\mathrm{A}=$ anterior, $\mathrm{P}=$ posterior, $\mathrm{H}=$ head, $\mathrm{F}=$ feet. 


\section{Global Journal of Otolaryngology}

\section{Results}

The study population consisted of 62 patients $(30$ males and 32 females) with a mean age of $51 \pm 17$ years and a range of 18-82 years. In 38 patients, both temporal bones met the predetermined criteria for inclusion in our study. 24 patients had only one of their temporal bones included. A total of 100 temporal bones (51 right and 49 left) were evaluated using the corresponding HRCT data sets. In the coronal plane the mean \pm standard deviation (SD) and the median \pm interquartile range (IQR) of $h_{c}$ was $4.4 \mathrm{~mm} \pm 1.2 \mathrm{~mm}$ and $4.15 \mathrm{~mm} \pm 1.6 \mathrm{~mm}$, respectively. In the sagittal plane the mean \pm SD and the median $\pm \mathrm{IQR}$ of $\mathrm{h}_{\mathrm{s}}$ was $2.66 \mathrm{~mm} \pm 1.38 \mathrm{~mm}$ and $2.5 \mathrm{~mm} \pm 1.9 \mathrm{~mm}$, respectively. The heights in coronal and sagittal planes ranged from $2.0-7.7 \mathrm{~mm}$ and $0.5-6.3 \mathrm{~mm}$, respectively. The frequencydistribution histograms demonstrate that both $\mathrm{h}_{\mathrm{c}}$ and $\mathrm{h}_{\mathrm{s}}$ display a unimodal distribution that resembles that of a normal cure, with the sagittal values resembleing a truncated normal curve with a right-skewed distribution (Figure 2). The Shapiro-Wilk Test of Normality showed that the $h_{c}$ values do not significantly deviate from the normal distribution $(p=0.203)$, whereas the $h_{s}$ values are significantly deviating from the normal distribution $(\mathrm{p}=0.005)$. However, further analysis of $\mathrm{h}_{\mathrm{s}}$ values using Kolmogorov-Smirnov test showed that there is no significant deviation from the truncated normal distribution $(\mathrm{p}=0.11)$. A box-plot depicting the spread as well as $95 \%$ confidence interval for both coronal and sagittal plane measurements is displayed in Figure 3.
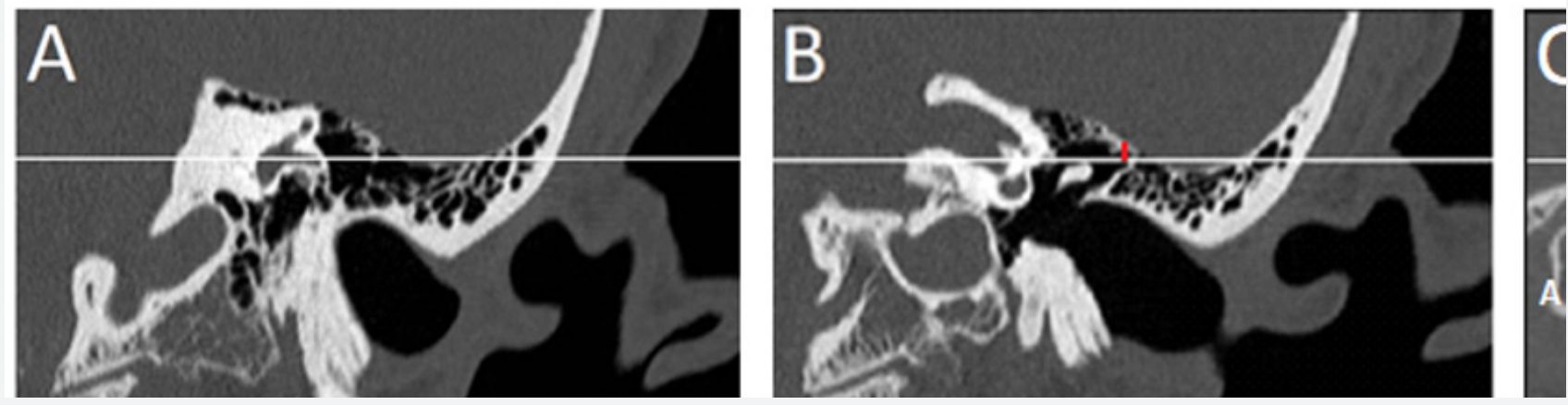

Figure 1: A. Horizontal line (white) through the plane of the lateral semicircular canal on a coronal image. B. Vertical line (red) perpendicular to the plane of the lateral semicircular canal used to measure the lowest coronal height of the tegmen tympani $\left(h_{c}\right)$. C. Horizontal line through the plane of the lateral semicircular from the coronal images. Vertical linerepresenting the lowest sagittal height of the tegmen tympani $\left(h_{s}\right) . A=$ anterior, $\mathrm{P}=$ posterior, $\mathrm{H}=$ head, $\mathrm{F}=$ feet.

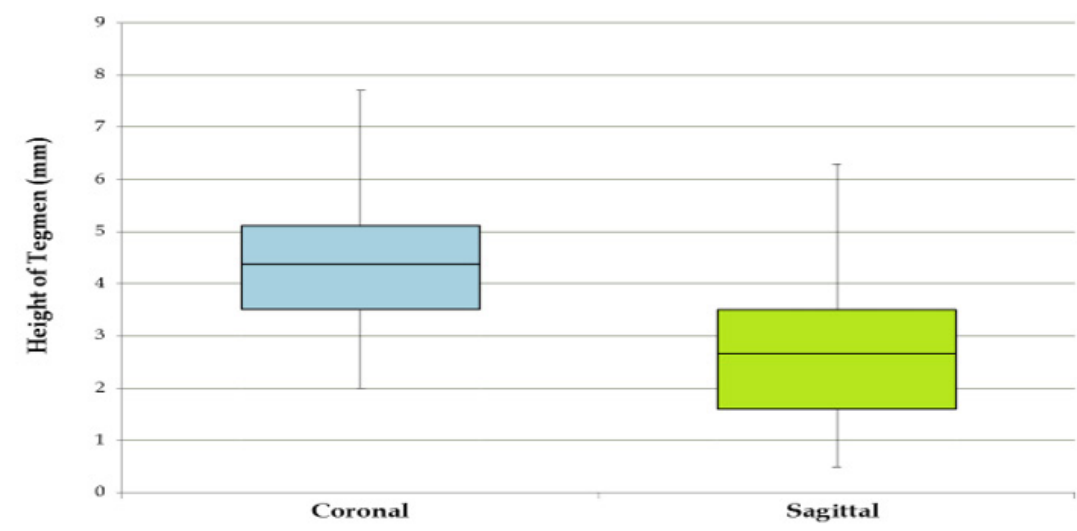

Figure 3: Box-plot depicting the spread as well as $95 \%$ confidence interval for both coronal (left) and sagittal (right) plane measurements.

\section{Discussion}

Identifying the location of key anatomic structures such as the tegmen tympani, facial nerve, and sigmoid sinus is a critical step in performing a safe mastoidectomy. The final location of these structures depends on temporal bone development. At birth, the mastoid antrum is well developed at about $1-1.5 \mathrm{~cm} 2$ in size [5]. Rapid pneumatization in the first year of normal development results in the mastoid cells increasing in size to about 3.5$5 \mathrm{~cm} 2$ followed by linear growth until age six $\left(1-1.2 \mathrm{~cm}^{2} /\right.$ year), and then a slower increase to the final adult size at puberty $(\sim 12 \mathrm{~cm} 2)[5]$. Patients with low-lying tegmens may represent those with insufficient mastoid development. The underlying etiology of insufficient mastoid development is debated in the literature with several authors suggesting a hereditary theory where mastoid aeration is genetically determined [6-9]. Other authors favor an environmental etiology, which suggests that chronic inflammation and Eustachian tube dysfunction during the early years of development predisposes to insufficient mastoid development [10,11]. 
A thorough understanding of the shape of the tegmen is important when performing surgery in the mastoid and middle ear [3]. A low-lying tegmen plate may increase the risk of breaching the middle cranial fossa when surgically exploring the epitympanum or mastoid. This may result in dural injury, with possible CSF leak and/or neural tissue damage. In their retrospective review of 356 temporal bones in 178 patients who underwent middle ear or mastoid surgery, Karaca et al. found that $26 \%$ of ears ( $n=94$ ) had low-lying tegmens and $14 \%$ of the 178 patients had bilateral low-lying tegmens [12]. HRCT of the temporal bones can provide crucial information regarding the anatomy of the temporal bone and the extent of disease which is not visible on clinical examination alone [13]. The degree of ventilation/opacification of the middle ear, erosion of the ossicular chain, dehiscence of the tegmen, and status of the facial nerve is some of the clinically valuable information that can be gathered from a good quality CT scan of the temporal bones1. Determining the height of the tegmen tympani also has practical implications relating to surgical access and may influence the surgical approach used. This is particularly useful when deciding whether an intact canal vs. canal wall down mastoidectomy should be performed.

In this study we propose the LSCC as a useful and constant landmark when measuring the height of the tegmen tympani on HRCT images of the temporal bones. It is an important surgical landmark which is easily identifiable when performing any mastoid surgery. Even if there is a fistula into the LSCC, the canal itself is seldom obliterated and is almost always readily identifiable on HRCT. Rarely is the LSCC malformed. The external ear canal may not be as useful a radiologic landmark, due to its variability in size and shape. In their series of 50 patients who underwent chronic otitis media surgery, Karatag et al. showed that, in patients where the dura was exposed as a complication of surgery $(n=12)$, the tegmen height was lower than in patients without dural exposure $(n=38)$ [2]. In this study, the tegmen height was measured as the distance between the lowest point on the tegmen and the spine of Henle. The spine of Henle is not an adequate nor optimal radiological landmark to measure the height of the tegmen tympani, as it is extremely variable, not obviously identified on HRCT imaging and, especially in patients who have had surgery on their ear canals or mastoid bones, is often non-existent.

Makki et al. investigated slope and shape variances of the tegmen and classified surgical risks based on the degree of convexity of the tegmen and how low the dura is in relation to the highest portion of the tegmen in the sagittal and coronal planes [3]. Their classification system stratified groups as low, intermediate, or high for potential risk of recurrence/tegmen penetration according to the distance between the tegmen and the superior semicircular canal (SSC) in the coronal plane and the tegmen and external auditory canal (EAC) in the sagittal plane. Surgically, the superior semicircular canal is not often sought out in mastoid surgery. To date, their classification has not been widely adopted amongst clinicians. The use of the external ear canal is understandable and to the surgeon very practical, but it remains an extremely variable landmark. In this study we propose a simpler classification system, hopefully one that everyday clinicians may be more able and willing to use.

Our study measured the distance between the LSCC and the lowest point of the tegmen tympani on HRCT scans of patients who underwent temporal bone imaging as part of their otologic workup for SNHL. The main purpose of this study was to determine the frequency distribution of this distance in radiographically normal temporal bones and to calculate the expected population averages and standard deviations. Our results indicate that, in a population of radiographically normal temporal bones, both the coronal and sagittal height of the lowest point of the tegmen tympani with respect to the LSCC $\left(h_{c}\right.$ and $h_{s}$ ) demonstrate a unimodal normal distribution. This implies that there is basically one average height in the coronal $(4.4 \mathrm{~mm} \pm 1.2 \mathrm{~mm})$ and sagittal $(2.66 \mathrm{~mm} \pm 1.38 \mathrm{~mm})$ planes, with a normally distributed range of variation around this value, rather than 2 or 3 common heights. The $h_{s}$ values demonstrated a prominent right-skewed distribution that deviatiate from a normal popultation when the Shapiro-Wilk Test of Normality was used $(\mathrm{p}=0.005)$. One explanation for this is that the relatively small mean values of $h_{s}$ resulted in clustering of the mean values around zero and resulted in truncation of the left side of an otherwise normal distribution. Applying Kolmogorov-Smirnov analysis to the $h_{s}$ values showed that there is no significant deviation from a truncated normal distribution $(p=0.11)$. The reason we chose the LSCC as the reference point for our measurements is because it is a stable radiological landmark, which is distinct, easily measurable and not often obliterated by diseases of the temporal bone or by surgery. Based on our results we propose a classification system where tegmen tympani heights bellow $4.5 \mathrm{~mm}$ on coronal measurements and $2.5 \mathrm{~mm}$ on sagittal measurements be considered "low" (type A) whereas tegmen tympani heights above these parameters be considered "high" (type B). This is based on the 50th percentile identified for tegmen heights in our population. Our cut-offs were rounded to the nearest $0.5 \mathrm{~mm}$ because mastoidectomy surgical burrs are typically commercially available only in $0.5 \mathrm{~mm}$ increments.

\section{Conclusion}

The height of the tegmen tympani at its lowest point with respect to the LSCC follows a unimodal normal distribution in the coronal and sagittal planes. We propose the LSCC as a useful and constant landmark when measuring the height of the tegmen tympani on HRCT images of the temporal bones. The LSCC is an important surgical landmark and a stable radiological landmark. Our technique establishes a reliable method to radiologically assess tegmen tympani height using the LSCC as a reference point. This system allows one to compare patient anatomy to a normal reference range and may help surgical planning. Future research examining possible correlations between tegmen 
tympani type and intraoperative complications is required to add clinical relevance to the proposed classification scheme.

\section{References}

1. Yates PD, Flood LM, Banerjee A, Clifford K (2002) CT scanning of middle ear cholesteatoma: What does the surgeon want to know? Br J Radiol 75(898): 847-852.

2. Karatag 0, Guclu 0, Kosar S, Derekoy FS (2014) Tegmen height: Preoperative value of $\mathrm{CT}$ on preventing dural complications in chronic otitis media surgery. Clin Imaging 38(3): 246-248.

3. Makki FM, Amoodi HA, van Wijhe RG, Bance M (2011) Anatomic analysis of the mastoid tegmen: Slopes and tegmen shape variances. Otology \& Neurotology 32(4): 581-588.

4. Marchioni D, Bonali M, Alicandri Ciufelli M, Rubini A, Pavesi G, et al. (2014) Combined approach for tegmen defects repair in patients with cerebrospinal fluid otorrhea or herniations: Our experience. Journal of neurological surgery.Part B, Skull base 75(4): 279.

5. Cinamon U (2009) The growth rate and size of the mastoid air cell system and mastoid bone: A review and reference. European Archives of Oto-Rhino-Laryngology 266(6): 781-786.

6. Sade J, Hadas E (1979) Prognostic evaluation of secretory otitis media as a function of mastoidal pneumatisation. Arch Otorhinolaryngol

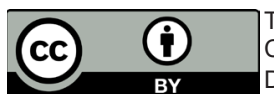

This work is licensed under Creative

Commons Attribution 4.0 License

DOI: 10.19080/GJO.2018.16.555944
225(1): 39-44.

7. Diamant M, Rubensohn G, Walander A (1958) Otosalpingitis and mastoid pneumatization. Acta Otolaryngo 149(1): 381-388.

8. Shea JJ (1971) Autoinflation treatment of serous otits media in children. The Journal of Laryngology \& Otology 85(12): 1254-1258.

9. Lindeman P, Shea JJ (1980) Size of the mastoid air cell system in children with middle ear effusion. Laryngoscope 90(11): 1840-1844.

10. Tos M, Stangerup S (1984) Mastoid pneumatization in secretory otitis further support for the environmental theory. Acta Otolaryngol 98(2): 110-118.

11. Tos M, Stangerup S, Hvid G (1984) Mastoid pneumatization: Evidence of the environmental theory. Archives of Otolaryngology 110(8): 502507.

12. Karaca CT, Toros SZ, Noseri HK (2012) Analysis of anatomic variations in temporal bone by radiology. Int Adv Otol 8: 239-243.

13. Mahmutoglu AS, Çelebi I, Sahinoglu S, Çakmakçi E, Sözen E (2013) Reliability of preoperative multidetector computed tomography scan in patients with chronic otitis media. J Craniofac Surg 24(4): 14721476.

Your next submission with Juniper Publishers will reach you the below assets

- Quality Editorial service

- Swift Peer Review

- Reprints availability

- E-prints Service

- Manuscript Podcast for convenient understanding

- Global attainment for your research

- Manuscript accessibility in different formats ( Pdf, E-pub, Full Text, Audio)

- Unceasing customer service

Track the below URL for one-step submission https://juniperpublishers.com/online-submission.php 